\title{
Bilinear Signal Synthesis Using Polarization Diversity
}

\author{
Moeness G. Amin, Fellow, IEEE, and Yimin Zhang, Senior Member, IEEE
}

\begin{abstract}
Bilinear synthesis of nonstationary signals impinging on a multiantenna receiver has been recently introduced. The distinction in the spatial signatures of the sources provides a vehicle to reduce noise and source signal interactions in the time-frequency domain, and hence improves signal synthesis. In this letter, we utilize another form of diversity for enhanced source time-frequency signal representations. It is shown that cross-polarization antennas can be used to mitigate cross terms via simple polarization averaging.
\end{abstract}

Index Terms-Polarization, signal synthesis, time-frequency (t-f) distribution.

\section{INTRODUCTION}

$\mathbf{T}$ IME-FREQUENCY distributions (TFDs) have been found useful in the analysis and classification of nonstationary signals [1], [2]. In [3], it is shown that the array manifold can be used to improve syntheses of signals with rapid time-varying frequency characteristics. In essence, averaging TFDs across different array sensors trades off the spatial dimension for enhanced autosource TFDs. Spatial averaging mitigates the cross-source time-frequency $(\mathrm{t}-\mathrm{f})$ terms as well as reduces the noise contribution.

When the receiver is not equipped with an antenna array, or the array is of small aperture, the spatial averaging of TFDs proposed in [3] will no longer be effective or applicable. A possible alternative is to use cross-polarization antennas where the polarization dimension can be utilized to enhance $\mathrm{t}-\mathrm{f}$ signature estimation and subsequently leads to improved signal synthesis performance. The polarization-based $\mathrm{t}-\mathrm{f}$ signal synthesis can be used for a single as well as multiple antennas. In this letter, we restrict our discussion to the simple case of a single pair of cross-polarization antennas. The generalization to applications of multisensor receivers is straightforward and is addressed in [4].

Signal polarization properties have been commonly utilized in wireless communications and synthetic aperture radars [5], [6]. Distinct polarization signatures of different sources can be observed when the sources have different transmitter polarizations or distinct channel characteristics.

This letter is organized as follows. The signal model is presented in Section II. Section III proposes the polarization av-

Manuscript received May 6, 2003; revised June 6, 2003. This work was supported in part by the Office of Naval Research under Grant N00014-98-1-0176 and in part by the Defense Advanced Research Projects Agency under Grant MDA972-02-1-0022. The content of the information does not necessarily reflect the position or policy of the Government, and no official endorsement should be inferred. The associate editor coordinating the review of this manuscript and approving it for publication was Prof. Xiang-Gen Xia.

The authors are with the Center for Advanced Communications, Villanova University, Villanova, PA 19085 USA (e-mail: moeness.amin@villanova.edu; yimin@ieee.org).

Digital Object Identifier 10.1109/LSP.2003.822901 eraging for $\mathrm{t}-\mathrm{f}$ signal synthesis. The analogy between the proposed method and the array averaging is also considered. Numerical simulations are given in Section IV.

\section{SignAl MODEL}

The discrete-time data received at a cross-polarization antenna, which receives two orthogonal polarizations (e.g., vertical and horizontal polarizations), is expressed in the following vector format:

$$
\mathbf{x}(t)=\left[x^{[p]}(t) \quad x^{[q]}(t)\right]^{T}
$$

where ${ }^{[p]}$ and ${ }^{[q]}$ represent the two orthogonal polarizations, and $T$ denotes transpose.

The expression

$$
\begin{array}{r}
D_{x^{[i]} x^{[k]}(t, f)=} \int_{-\infty}^{\infty} \int_{-\infty}^{\infty} \phi(t-u, \tau) x^{[i]}\left(t+\frac{\tau}{2}\right) \\
\cdot\left(x^{[k]}\left(t-\frac{\tau}{2}\right)\right)^{*} e^{-j 2 \pi f t} d u d \tau
\end{array}
$$

defines the auto- $(i=k)$ and cross-polarized $(i \neq k)$ TFDs of the two polarizations, where $t$ and $f$ are the time and the frequency indexes, respectively, $\phi(t, \tau)$ is the time-frequency kernel [7], and ${ }^{*}$ denotes complex conjugate. Each of $i$ and $k$ takes either value of the polarization index $p$ or $q$. The auto- and cross-polarized TFDs can be combined to form the following $2 \times 2$ polarization TFD matrix:

$$
\begin{array}{r}
\mathbf{D}_{\mathbf{x x}}(t, f)=\int_{-\infty}^{\infty} \int_{-\infty}^{\infty} \phi(t-u, \tau) \mathbf{x}\left(t+\frac{\tau}{2}\right) \\
\cdot \mathbf{x}^{H}\left(t-\frac{\tau}{2}\right) e^{-j 2 \pi f t} d u d \tau
\end{array}
$$

where superscript ${ }^{H}$ denotes transpose conjugation. The diagonal entries of $\mathbf{D}_{\mathbf{x x}}(t, f)$ are the autopolarized TFDs, whereas the off-diagonal elements are cross-polarized TFDs.

Assume $L$ source signals $s_{l}(t), l=1, \ldots, L$, are incident on the antenna. The received data for each polarization is the linear combination of the same polarization components of the source signals and noise. That is,

$$
x^{[i]}(t)=\sum_{l=1}^{L} a_{l}^{[i]} s_{l}(t)+n^{[i]}(t), \quad i=p, q
$$

where $a_{l}^{[i]}$ represents the mixing coefficient of the $l$ th source along the $i$ th polarization, and $n^{[i]}(t)$ is the noise component at the same polarization. In the vector form, $\mathbf{x}(t)$ can be decomposed into the following terms:

$$
\mathbf{x}(t)=\mathbf{y}(t)+\mathbf{n}(t)=\mathbf{A} \mathbf{s}(t)+\mathbf{n}(t)=\sum_{l=1}^{L} \mathbf{a}_{l} s_{l}(t)+\mathbf{n}(t)
$$


where $\mathbf{a}_{l}=\left[\begin{array}{ll}a_{l}^{[p]} & a_{l}^{[q]}\end{array}\right]^{T}, \mathbf{A}=\left[\mathbf{a}_{1}, \ldots, \mathbf{a}_{L}\right], \mathbf{s}(t)=$ $\left[\mathrm{s}_{1}(t), \ldots, \mathrm{s}_{L}(t)\right]^{T}$, and $\mathbf{n}(t)=\left[n^{[p]}(t) n^{[q]}(t)\right]^{T}$. Because of the ambiguity with respect to the signal strength and the propagation attenuation, it is convenient to assume that $\left\|\mathbf{a}_{l}\right\|_{2}^{2}=2, l=1, \ldots, L$, and the propagation attenuation scalar is absorbed in $s_{l}(t)$. The noise elements are modeled as stationary and white complex Gaussian processes with zero mean and variance $\sigma^{2}$ in each polarzation, i.e.,

$$
E\left[\mathbf{n}(t+\tau) \mathbf{n}^{H}(t)\right]=\sigma^{2} \delta(\tau) \mathbf{I}_{2}
$$

where $\delta(\tau)$ is the Kronecker delta and $\mathbf{I}_{N}$ denotes the $N \times N$ identity matrix.

\section{Polarization AVERAging}

It is clear from Section II that the signal model of the crosspolarization antenna case is similar to that of a two-antenna array. The only difference is that the source polarization vector $\mathbf{a}_{l}$ is used in place of the source spatial signature, or steering vector. Accordingly, polarization averaging can be equally effective as spatial averaging in mitigating the TFD cross terms.

From (2) and (4), the autopolarization TFD of $x^{[i]}(t)$ is given by

$$
\begin{aligned}
D_{x^{[i]} x^{[i]}}(t, f)=\sum_{l=1}^{L} \sum_{m=1}^{L} a_{l}^{[i]}\left(a_{m}^{[i]}\right)^{*} D_{s_{l} s_{m}}(t, f) & \\
& +D_{n^{[i]} n^{[i]}}(t, f), \quad i=p, q
\end{aligned}
$$

where $D_{s_{l} s_{m}}(t, f)$ represents the autosource TFD (if $l=m$ ) or the cross-source TFD (if $l \neq m$ ). The presence of cross-source terms often obscures the true power localization over time and frequency.

Averaging the autopolarization TFDs over the two polarization branches yields

$$
\begin{aligned}
\overline{\mathrm{W}}(t, f)= & \frac{1}{2} \sum_{i=p}^{q} D_{x^{[i]} x^{[i]}}(t, f) \\
= & \sum_{i=1}^{L} \sum_{j=1}^{L}\left(\frac{1}{2} \sum_{i=p}^{q} a_{l}^{[i]}\left(a_{m}^{[i]}\right)^{*}\right) D_{s_{l} s_{m}}(t, f) \\
& +\frac{1}{2} \sum_{i=p}^{q}\left[D_{n^{[i]} n^{[i]}}(t, f)\right] \\
= & \sum_{i=1}^{L} \sum_{j=1}^{L}\left(\frac{1}{2} \mathbf{a}_{m}^{H} \mathbf{a}_{l}\right) D_{s_{l} s_{m}}(t, f) \\
& +\frac{1}{2} \sum_{i=p}^{q}\left[D_{n^{[i]} n^{[i]}}(t, f)\right] .
\end{aligned}
$$

In (8), $\mathbf{a}_{m}^{H} \mathbf{a}_{l}$ is the inner product of the polarization signatures $\mathbf{a}_{m}$ and $\mathbf{a}_{l}$. Define the polarization correlation coefficient

$$
\beta_{l m}=\frac{1}{2} \mathbf{a}_{m}^{H} \mathbf{a}_{l} .
$$

Accordingly, (8) can be expressed as

$$
\overline{\mathrm{W}}(t, f)=\sum_{i=1}^{L} \sum_{j=1}^{L} \beta_{l m} D_{s_{l} s_{m}}(t, f)+\frac{1}{2} \sum_{i=p}^{q}\left[D_{n^{[i]} n^{[i]}}(t, f)\right] .
$$

The above equation shows that $\overline{\mathrm{W}}(t, f)$ is a linear combination of the auto- and cross-polarization TFDs of all signal arrivals. It is straightforward to show that for the $l$ th and the $m$ th sources

$$
\begin{array}{rlrl}
\left|\beta_{l m}\right| \leq 1, & & \text { if } l \neq m \\
\beta_{l m} & =1, & & \text { if } l=m
\end{array}
$$

indicating that the constant coefficients in (10) for the autopolarization TFDs are always greater than, or at least equal to, those for the cross-polarization TFDs. For sources with distinct polarizations, $\left|\beta_{l m}\right| \ll 1$, leading to significant suppression of cross terms, and thereby enhancing the signal signature estimation.

An interesting case arises when two signals have orthogonal polarization signatures, i.e., $\beta_{l m}=0$ for $l \neq m$. In this case, the cross terms between these two source signals will be entirely eliminated and only the autoterms will be maintained.

The $\mathrm{t}-\mathrm{f}$ kernel in (2) and (3), which introduces temporal averaging of the local autocorrelation functions at consecutive time samples, can be selected to reduce the TFD noise effect for the single antenna case, as discussed in [8] and [9]. However, even without kernel smoothing, the polarization averaging in (10), similar to spatial averaging [10], decreases the noise variance and its interaction with the signal components beyond that achieved in a single antenna (polarization) case. Once the polarization averaging is performed and the $\mathrm{t}-\mathrm{f}$ signature is identified, we can then proceed with the bilinear syntheses using the methods described in [2].

It is noted that, although the model used allows for $L$ source signals to be present, there are only two dimensions of polarization diversity for a single cross-polarization antenna. Therefore, when $L>2$, while the cross terms between different source signals can still be substantially mitigated, it becomes impossible to completely eliminate all the cross terms unless more sensors are used.

\section{Simulation Results}

In this section, we provide computer simulations to demonstrate the improvement gained by the proposed technique in the reduction or elimination of cross terms and signal synthesis. Two high-order frequency modulated signals are considered on a dual-polarization dipole. Their polarizations are assumed to be orthogonal, with the following mixing matrix:

$$
\mathbf{A}=\left[\begin{array}{rr}
1 & 1 \\
1 & -1
\end{array}\right]
$$

The length of the signal sequence is set to $N=256$. The additive noise is zero mean, Gaussian distributed, and white. The input $\mathrm{SNR}$ is $3 \mathrm{~dB}$.

With the presence of high-level noise and close $t-f$ signatures, it is very difficult to identify these $t-f$ signatures when only a single-polarization sensor is used. Fig. 1 shows the extended discrete-time Wigner-Ville distribution (EDTWVD) [11] of the 


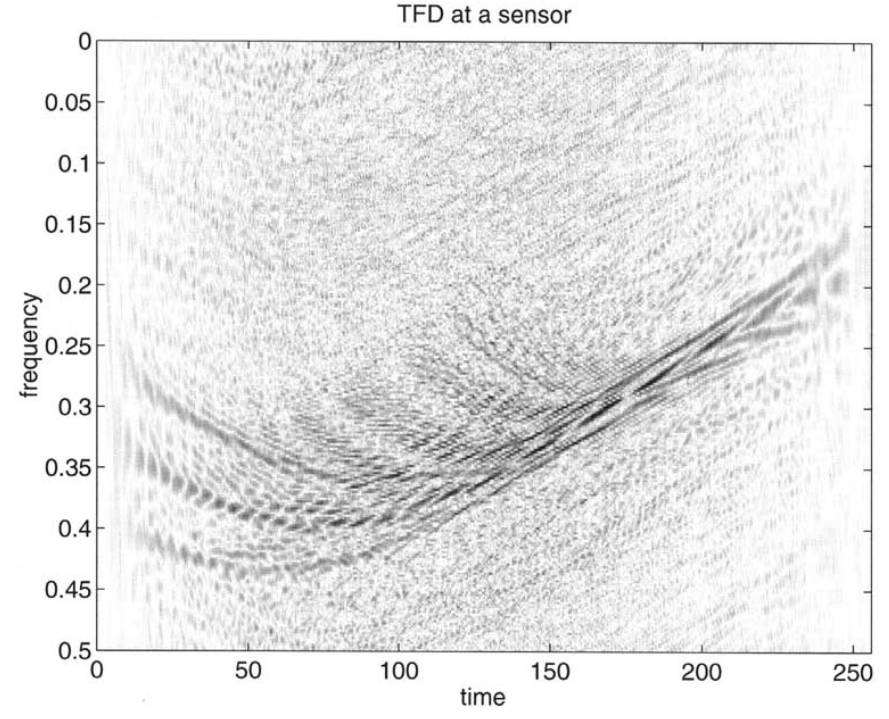

Fig. 1. EDTWVD computed from the signal received at the vertical polarization antenna.

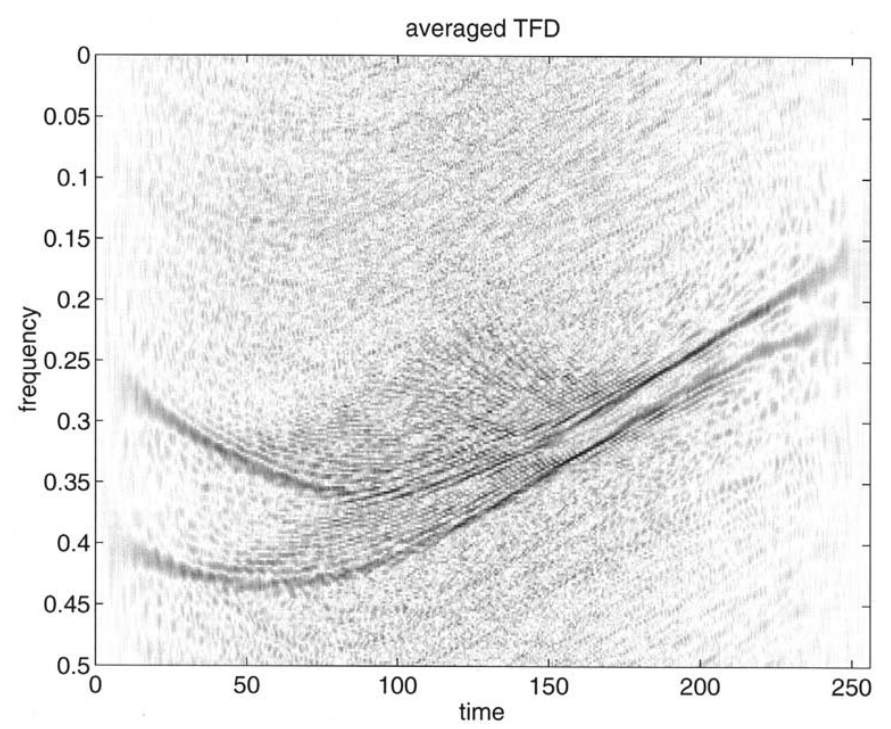

Fig. 2. EDTWVD averaged over two polarizations.

data received at the vertically polarized antenna. However, as evident from Fig. 2, the $t-f$ signatures of the two signals can be revealed when polarization averaging is applied. Fig. 2 shows that the cross term between the two signals is completely eliminated and that the variance of noise terms is reduced. Masking the first signal and applying standard signal synthesis techniques yield a high-quality signal recovery. Fig. 3 shows the TFD of the synthesized signal waveform of the first signal.

\section{CONCLUSION}

Polarization averaging allows effective cross-term reduction and autoterm enhancement, aiding source time-frequency

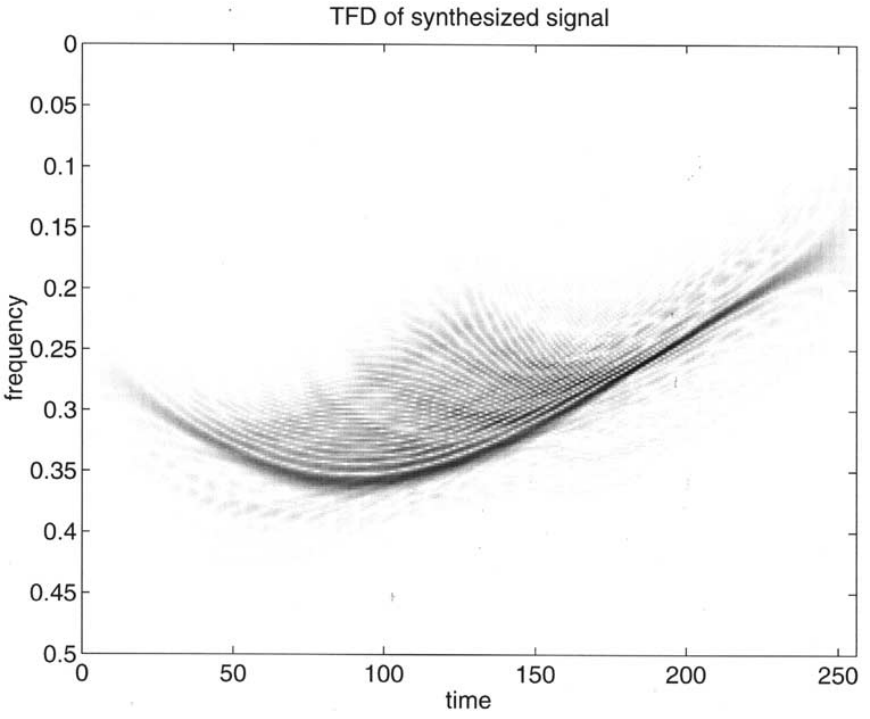

Fig. 3. EDTWVD of the synthesized waveform of the first signal.

signature estimations and waveform recovery. Averaging TFDs across polarizations can be performed concurrently with TFD averaging across the array, thereby utilizing both spatial and polarization diversity in syntheses of nonstationary signals. However, polarization averaging can be applied alone if the difference in the source spatial signatures is insufficient for cross-term reduction, or the receiver is not equipped with antenna arrays.

\section{REFERENCES}

[1] B. Boashash, "Time-frequency signal analysis," in Advances in Spectrum Analysis and Array Processing, S. Haykin, Ed. Englewood Cliffs, NJ: Prentice-Hall, 1990, vol. 1, ch. 9, pp. 21-68.

[2] F. Hlawatsch and G. Boudreaux-Bartles, "Linear and quadratic time-frequency signal representations," IEEE Signal Processing Mag., vol. 9, pp. 21-68, Apr. 1992.

[3] W. Mu, M. G. Amin, and Y. Zhang, "Bilinear signal synthesis in array processing," IEEE Trans. Signal Processing, vol. 51, pp. 90-100, Jan. 2003.

[4] Y. Zhang, M. G. Amin, and B. A. Obeidat, "The spatial polarimetric time-frequency distributions and their applicaiton to direction-of-arrival estimation," Proc. SPIE, vol. 5205, Aug. 2003.

[5] J. D. Gibson, The Mobile Communications Handbook. New York: CRC, 1996.

[6] F. Sadjadi, "Improved target classification using optimum polarimetric SAR signatures," IEEE Trans. Aerosp. Electron. Syst., vol. 38, pp. 38-49, Jan. 2002.

[7] L. Cohen, Time-Frequency Analysis. Upper Saddle River, NJ: Prentice-Hall, 1995

[8] M. G. Amin, "Minimum-variance time-frequency distribution kernels for signals in additive noise," IEEE Trans. Signal Processing, vol. 44, pp. 2352-2356, Sept. 1996.

[9] L. J. Stankovic, "Analysis of noise in time-frequency distributions," IEEE Signal Processing Lett., vol. 9, pp. 286-289, Sept. 2002.

[10] Y. Zhang, A. R. Lindsey, and M. G. Amin, "Combined synthesis and projection techniques for jammer suppression in DS/SS communications," in Proc. ICASSP, Orlando, FL, May 2002.

[11] J. Jeong and W. Williams, "Time-varying filtering and signal synthesis," in Time-Frequency Signal Analysis-Methods and Applications, B. Boashash, Ed. White Plains, NY: Longman Cheshire, 1995. 